\title{
Article
}

\section{Delivering motivational interviewing early post stroke: standardisation of the intervention}

Patel, Kulsum, Auton, Malcolm Frederick, Watkins, Caroline Leigh, Sutton, Chris J, Benedetto, Valerio, Hackett, Maree, Holland, EmmaJoy and Lightbody, Catherine Elizabeth

Available at http://clok.uclan.ac.uk/36233/

Patel, Kulsum, Auton, Malcolm Frederick ORCID: 0000-0002-8173-8159, Watkins, Caroline Leigh ORCID: 0000-0002-9403-3772, Sutton, Chris J ORCID: 0000-0002-6406-1318, Benedetto, Valerio ORCID: 0000-0002-4683-0777, Hackett, Maree ORCID: 0000-0003-1211-9087, Holland, Emma-Joy ORCID: 0000-0003-3029-7573 and Lightbody, Catherine Elizabeth ORCID: 0000-00015016-3471 (2022) Delivering motivational interviewing early post stroke: standardisation of the intervention. Disability and Rehabilitation, 44 (14). pp. 3453-3458. ISSN 0963-8288

It is advisable to refer to the publisher's version if you intend to cite from the work. http://dx.doi.org/10.1080/09638288.2020.1864035

For more information about UCLan's research in this area go to http://www.uclan.ac.uk/researchgroups/ and search for < name of research Group>.

For information about Research generally at UCLan please go to http://www.uclan.ac.uk/research/

All outputs in CLoK are protected by Intellectual Property Rights law, including Copyright law. Copyright, IPR and Moral Rights for the works on this site are retained by the individual authors and/or other copyright owners. Terms and conditions for use of this material are defined in the policies page. 
Delivering motivational interviewing early post stroke: standardisation of the intervention

\section{Authors:}

Kulsum Patel.* University of Central Lancashire, Preston, UK. kpatel@uclan.ac.uk Malcolm F. Auton. University of Central Lancashire, Preston, UK. mfauton@uclan.ac.uk Caroline L. Watkins. University of Central Lancashire, Preston, UK. clwatkins@ uclan.ac.uk Christopher J. Sutton. The University of Manchester. chris.j.sutton@ manchester.ac.uk Valerio Benedetto. University of Central Lancashire, Preston, UK. vbenedetto@uclan.ac.uk Maree L. Hackett. The George Institute for Global Health, Australia. mhackett@georgeinstitute.org.au Emma-Joy Holland. University of Central Lancashire, Preston, UK. eholland1 @uclan.ac.uk Catherine E. Lightbody. University of Central Lancashire, Preston, UK.

celightbody@uclan.ac.uk

* corresponding author

Word count: 2982 


\begin{abstract}
Background: We applied Motivational Interviewing (MI) techniques, early after stroke, to facilitate psychological adjustment to life post-stroke. In our trial, MI-plus-usual-care increased the likelihood of normal mood at 3-months post-stroke, compared to usual-care alone. Whilst appropriate training, manuals, and supervision may increase adherence to core principles of this complex intervention, unintended variability in implementation inevitably remains. We aimed to explore the impact of variability on participant outcome.
\end{abstract}

Methods: Using our trial data (411 participants), we explored variation in MI delivery, examining: therapist characteristics (stroke care expertise/knowledge, psychology training); MI content (fidelity to MI techniques assessed with Motivational Interviewing Treatment Integrity code, describing therapist behaviours as MI-consistent, MI-neutral or MIinconsistent); and MI dose (number/duration of sessions).

Results: The four MI therapists (two nurses/two psychologists) had varying expertise and MI delivery. Across therapists, mean average session duration ranged 29.5-47.8 minutes. The percentage of participants completing the per-protocol four sessions ranged $47 \%-74 \%$. These variations were not related to participant outcome. There were uniformly high frequencies (>99\%) of MI-consistent and MI-neutral interactions, and low frequencies (<1\%) of MIinconsistent interactions.

Conclusions: Variation in therapist characteristics and MI dose did not affect participant outcome. These may have been tolerated due to high fidelity to MI principles.

\title{
Keywords
}


Motivational Interviewing, complex interventions, stroke, depression, therapist effects

\section{Introduction}

Motivational Interviewing (MI) is a talk-based therapy, originally developed to help people with addictions [1]. Using specific MI person-centred techniques, MI therapists support clients' self-efficacy to build confidence and develop motivation and readiness to change. MI has been modified to be delivered to stroke survivors early after their stroke and the technique used to promote self-efficacy and facilitate adjustment to life after stroke [2]. Given the prevalence [3] and associated negative impacts [4,5] of post-stroke psychological problems, such as depression, and the lack of psychological support available for stroke survivors [6], it is important to consider psychological interventions for this patient population to address this need [6]. In an evaluation of its effectiveness, the results of a randomised controlled trial (RCT) indicated that MI early post-stroke had a beneficial effect on mood: people who received up to four weekly sessions of MI early after their stroke in addition to usual care were less likely to have low mood at three months post-stroke, compared with participants who received usual care alone [2]; this effect was maintained at 12 months post-stroke [7].

MI is a complex intervention to standardise; its effectiveness is assessed across multiple facets [8]. Talk-based therapies will inevitably vary in staff provision and engagement of participants [9]. To reduce variability, some standardisation of complex interventions is necessary within a trial setting, usually by provision of a protocol or manual for therapists to follow. Fidelity to the manual is measured during the trial $[10,11]$. It is important to consider treatment fidelity, the degree to which the intervention is delivered as intended, to ensure validity of the trial's findings so that any evidence-based treatments can be effectively 
disseminated [12]. Whilst appropriate training and the use of intervention manuals within trials allow reproducibility and maintain consistency of intervention delivery within and across sites, therapists and individual participants, some variability in implementation is inevitable. The Medical Research Council (MRC) has cautioned against strict adherence to implementation protocols to allow for local variation [13], but it can be difficult to decide which intervention component(s) should be subject to flexibility and which should be standardised $[13,14]$. Fidelity to interventions should be assessed on core intervention components, while flexibility in less central components can be modulated to achieve better fit [15]. However, it is often difficult to know what the core components are for complex interventions [13]. Furthermore, therapists who are more skilled may vary (tailor) delivery of an intervention to the participant, as they do in clinical practice, as opposed to adhering to a suggested less flexible approach [16]. This may be considered more acceptable than 'drifting' where deviations from the intervention are unintentional [17].

In the RCT of MI post-stroke [2], the delivery of the intervention was standardised through robust protocols/manuals, group training and consistent supervision of the MI therapists delivering the intervention. Variability in intervention delivery was permitted in order to meet participant needs (e.g. MI therapists were asked to deliver one-hour sessions, but this might vary according to participant fatigue). However, in complex interventions, small variations in some or all aspects of delivery, content, duration or intensity could result in large differences in outcome, while larger variations in other aspects could have little impact on outcome [8].

We aimed to explore the variation in the following aspects of the MI intervention used in the MI post-stroke RCT [2]: therapist characteristics (experience and professional background of 
MI therapists); MI content (adherence to principles of MI); and MI dose (number and duration of MI sessions).

\section{Methods}

\section{Trial summary}

This article reports on an evaluation of a MI intervention embedded within a RCT described previously [2,7]. In brief, patients admitted to hospital with acute stroke, who were aged over 18 years and did not have severe cognitive or communication problems preventing them from engaging in a conversation, were invited to participate. 411 consenting participants were randomised early (up to 28 days) after stroke to usual care ( $\mathrm{n}=207)$ or MI in addition to usual care $(n=204)$. The mood of all participants was assessed at baseline, 3 and 12 months using the General Health Questionnaire (GHQ-28 [18]: range 0-28). The primary outcome in the trial was participant mood (low $\geq 5$; normal $<5$ ) at three months. The trial was approved by the local research ethics committee (Ref: 99/187) and by the Research and Development department in the Trust.

\section{Intervention and standardisation}

Four therapists were trained together in MI by a commercial MI trainer through presentations, workshops and role play. Training comprised two blocks of two full day sessions held two weeks apart, followed by up to 10 practice sessions with volunteer patients from a stroke review clinic. This resulted in approximately 50 hours of training. Audio recordings of sessions with practice patients were reviewed by an MI-trained clinical psychologist until each therapist was judged competent, that is $90 \%$ MI-adherent as assessed with the Motivational Interviewing Treatment Integrity code (MITI 3.1.1 [19]), to conduct MI sessions with trial participants. 
The intervention was standardised as much as necessary using a detailed protocol. The four trained MI therapists were allocated participants by random permuted blocks. MI sessions were arranged and delivered face-to-face and individually by the allocated therapist, in a quiet room in hospital or at the participant's home, depending on participant choice if postdischarge. It was intended that each participant randomised to receive MI would participate in four one-hour sessions, each held one week apart.

The first session followed a structured framework where the therapist set the agenda so the participant talked about their adjustment to stroke and current concerns [20]. Subsequent sessions were largely participant-led but the remit of the therapists was to adhere to the spirit of MI delivery and avoid MI-inconsistent behaviour. MI-consistent behaviours were used to elicit participants' post-stroke concerns and help them to explore and use their own resources to address these concerns.

Fidelity to MI was assessed throughout the trial by the clinical psychologist, who did not deliver MI during the trial, using the recommended MITI 3.1.1 coding [19]. The MITI has been shown to be reliable and valid [21]. The MITI therapist codes were used to categorise therapist utterances (a complete concept) as MI-consistent or MI-inconsistent. Key elements of an MI-consistent approach are open questions, simple reflections (therapist repeats back explicit content of what the participant has said) and complex reflections (therapist includes the participant's unspoken meanings, feelings or intentions); other MI-consistent codes include affirming, summarising, and advising with permission. MI-inconsistent codes include confronting, warning, arguing, negating, and advising without permission. A third category, MI-neutral, was used for utterances that were not classified as MI-consistent or MI- 
inconsistent, including fillers and closed questions. The MITI also includes global ratings to assess a therapist's overall adherence to MI principles within a session. Global ratings assess therapist levels of empathy, MI spirit and acceptance on a 7-point scale, with a rating score of at least 6 required for MI competency.

As a quality assurance, measure of treatment fidelity and support feature, the therapists were supervised and mentored during the trial by the clinical psychologist during monthly team meetings. There was also regular review of voice/video files and logs from MI sessions, oneto-one supervision sessions, and additional informal support as required. Therapists had to record all sessions and maintain session logs; these were available to the clinical psychologist and used to assess and maintain fidelity and quality of MI delivery through regular feedback to the therapists.

\section{Sampling}

All voice files $(n=693)$ where data on dose and participant outcome were available were used in the analysis. In the RCT, a randomly selected $20 \%$ sample of voice files $(n=137)$ had been transcribed for fidelity assessment during the trial. The data for this study were elicited from 60 of the transcribed files, which was chiefly determined on pragmatic grounds, but was deemed a sufficiently large number for this study, and similar integrity subsamples have been reported elsewhere $[21,22]$. These transcripts were purposively sampled to maximise the diversity of: therapist; participants' age and sex; stroke severity assessed by the Barthel Index [23]; presence or absence of low mood at baseline, and session number, whilst ensuring that each participant was only selected once.

\section{Intervention components}




\section{Therapist characteristics}

The sex, training, caseload and stroke and psychology knowledge of the therapists, which may have influenced the delivery of the intervention, are described narratively.

\section{Content}

The delivery of the intervention was assessed using the MITI codes and global ratings, by the supervising clinical psychologist and two of the MI therapists (who may have assessed their own sessions). The clinical psychologist and one of the two therapists each coded the therapist utterances and scored the global ratings in the aforementioned 60 transcripts, with any disagreements in coding/ratings discussed and a consensus agreed. From these we collated the data for each therapist's performance.

\section{Dose}

The duration and number of sessions (maximum of four), which have been previously described as measures of dose [24], were obtained from the therapists' session logs.

\section{Analysis}

Therapist was used as the unit of analysis. We used descriptive statistics to illustrate differences between therapists in terms of therapist characteristics, session features, fidelity to MI, and univariable logistic regression to investigate sessional factors (therapist, number of sessions, participant's average session duration) potentially impacting on participant outcome. A successful outcome for this study was considered to be a participant having normal mood (GHQ-28 score <5) at three months post-stroke.

\section{Results}


Differences in therapist characteristics, session content and session dose across the therapists are shown in table 1.

\section{Therapist characteristics}

Two female therapists were nurses: one was a senior member of the stroke clinical team, the other was from the research team; both had extensive knowledge of stroke. There was one male therapist with a background in psychology (non-clinical) with a fair knowledge of stroke. The fourth therapist was female and a recent psychology graduate recruited to the research team specifically for the post, with limited knowledge of stroke. One therapist left the project prematurely and was not replaced. Another therapist delivered considerably fewer sessions due to maternity leave. Both these therapists completed sessions with participants on their caseload prior to leaving the study.

\section{Content}

The four therapists were rated at a 'proficient' level and global quality ratings showed little variation between therapists as sessions were consistently rated high for empathy, MI spirit and acceptance. There was variation in how each therapist achieved that proficiency and fidelity; for example, there were different patterns in the use of neutral responses (e.g. utterances such as 'mmm' or 'yeah'), closed questions and reflections (table 2). Less than 1\% of total utterances for each therapist were MI-inconsistent.

\section{Dose}

Except for Therapist C, who delivered fewer sessions overall, there was little difference between therapists in the percentage of their participant case load who received four sessions. 
Mean participant-average session duration varied from 30 to 48 minutes between the four therapists.

[Table 1 near here]

[Table 2 near here]

Observed therapist-specific success rates of MI (normal mood at three months) varied from $38 \%$ to $62 \%$, although differences were not significant $(\mathrm{p}=0.26)$. Likewise, success was not related to either the number of sessions $(\mathrm{p}=0.21)$, or the participant's average session duration $(\mathrm{p}=0.58)$.

\section{Discussion}

This study found there was moderate variation in session duration between therapists, and little variation in number of sessions and fidelity to MI and quality of the interactions. These are discussed in the context of the study and application to clinical practice.

The largest differences between the therapists was in the mean of the average duration of a participant's sessions. Assuming there is enough time for quality discussion, and as most change talk has been found to occur in the final fifth of sessions regardless of session duration [25], session duration seems to be of relatively little importance. While sessions of greater than 20 minutes duration have been shown to be more effective than shorter sessions [26], the shortest mean participant-average session duration by therapists in our study was 30 minutes. Session duration might therefore be considered a 'green light' adaptation, that is, a safe adaptation that does not compromise the intervention and allows better fit for the individuals involved or the intervention setting [27]. 
We found little variation between the therapists in the average number of sessions which participants completed. However, one therapist (C) had a lower percentage of participants completing all four sessions than the others, but therapist $\mathrm{C}$ treated too few participants to infer any impact on outcome. There is limited evidence for the optimum number of sessions of MI. Two or more sessions have been shown to be marginally more effective than one, but even one session has been shown to produce a positive outcome in some applications of MI [26]. The number of MI sessions might therefore be considered a peripheral component of the intervention, in which variation is acceptable. However, further exploration of this is required in a stroke population, including how this might vary with other factors, such as communication, functional, or cognitive abilities. Further exploration of the dose of MI is also required: although operationalised in this study as the number and duration of sessions, there may be other factors constituting dose, such as degree of therapeutic alliance [28].

Although we did find variation between therapists in the application of MI as assessed using the MITI, three important consistencies were evident: high levels of MI-consistent interactions, the rare occurrence of MI-inconsistent interactions, and high global ratings of MI treatment integrity. Given the minor variations in session factors between therapists, MI consistency may explain the positive impact on mood, and therefore may represent the core intervention component that should be adhered to. This finding is congruent with an earlier observation that a single MI-inconsistent behaviour (e.g. confrontation) was a reliable predictor of a negative outcome [29]. It is also compatible with two scoping reviews which concluded that avoiding MI-inconsistent interactions was the only factor that was reliably related to outcome [30,31]. The therapeutic environment with frequent MI-consistent behaviours and explicit avoidance of MI-inconsistent behaviours in our trial may be a result 
of the detailed protocol, standardised training, and supervision of the therapists by an experienced clinical psychologist.

Therapists potentially have an impact on the effectiveness of therapy or outcome. However, a review of health professionals carrying out MI concluded that their clinical role does not seem to have any significant impact on effectiveness [30]. Our trial of MI used therapists from inside and outside the health professions with no obvious variation in outcome, providing further evidence that the professional background and experience of MI therapists may not be a strong determining factor for the effectiveness of MI. Additionally, participant factors may have contributed to measured outcomes to a greater extent than therapist factors. It may also be that the relationship between the therapist and the patient (therapeutic alliance), which was not assessed in our study, is more important than the individual professional or participant characteristics when assessing the impact of the therapist on intervention effectiveness. However, we focused on therapist factors as these, unlike participant or therapist-participant relationship characteristics, are aspects that we can have some control over and may offer greater considerations for clinical application of the intervention. As such, therapist effects should be an integral, prospective consideration in trials of psychological therapy [32].

In order to increase validity and reduce confounding, researchers try to minimise variability in intervention delivery. Whilst this is important, it is less feasible to control for variability in clinical practice; hence it is important to know what factors are important and what measures should be implemented to control variability and increase adherence to core intervention principles, and what aspects can be allowed to vary, and tailored to the preference or needs of the therapist or patient. In the case of a complex intervention such as MI it is difficult to 
isolate a causal link between the intervention and outcome. We have shown that a good outcome is possible despite variation in some therapist and dose factors.

A limitation of this study is that the trial was of moderate size, involved only four therapists, and was not powered to detect potential therapist effects. Future trials with larger numbers of participants and therapists, designed to investigate which aspects of MI delivery (e.g. duration, number of sessions, content, therapeutic alliance) impact on the effectiveness of the intervention, and hence how MI in stroke care might be optimised, are required.

This study used the MITI version 3.1.1 which has since been revised, with the updated version describing some limitations of the older versions [33]. However, the limitations are generally related to the lack of identification of participant 'change talk' which is a key principle of MI, and the tool being unwieldy for use in clinical practice for supervision purposes. As our use of the tool was to determine therapist fidelity to MI techniques in research, the results reported here are unlikely to be affected by these limitations. Furthermore, the structural changes to MI published in 2012 [34] would make it difficult to revisit the data and conduct analysis using the updated version of the MITI. Thus, in future research, use of the most current version of the MITI would be necessary to allow an evaluation of its use in clinical practice in a stroke care setting.

Our results indicate that our MI intervention can tolerate some variation in peripheral aspects (therapist characteristics and dose as we have operationalised it) and still be effective when the core components are adhered to. However, it may be that other factors contribute to the dose of MI, and the number and duration of sessions are not the most important aspects of the intervention received by participants. Therefore, we suggest that future trials of MI may also 
achieve a positive outcome through the therapists delivering MI of high treatment integrity, following appropriate training and supervision. If MI is shown to be effective in larger-scale studies, the same principles should then be applied for use in clinical practice.

\section{Acknowledgement}

Maree Hackett holds a National Health and Medical Research Council of Australia (NHMRC) Career Development Fellowship. Valerio Benedetto is part-funded by the National Institute for Health Research Applied Research Collaboration North West Coast (NIHR ARC NWC). The views expressed are those of the author and not necessarily those of the NIHR or the Department of Health and Social Care.

\section{Declaration of competing interests}

The authors report no conflicts of interest.

\section{Data availability statement}

The data that support the findings of this study are available from the corresponding author, upon reasonable request.

\section{References}

1. Miller WR, Rollnick S. Ten things that motivational interviewing is not. Behavioural and Cognitive Psychotherapy. 2009;37(2):129-140.

2. Watkins CL, Auton MF, Deans CF, et al. Motivational interviewing early after acute stroke: a randomized, controlled trial. Stroke. 2007;38:1004-1009. 
3. Hackett M, Pickles K. Part I: frequency of depression after stroke: an updated systematic review and meta-analysis of observational studies. International Journal of Stroke. 2014;9(8):1017-25.

4. Willey JZ, Disla N, Moon YP, et al. Early depressed mood after stroke predicts long-term disability: the northern Manhattan stroke study (NOMASS). Stroke. 2010;41(9):1896-900.

5. Bartoli F, Lillia N, Lax A, et al. Depression after stroke and risk of mortality: a systematic review and meta-analysis. Stroke Research and Treatment. 2013;862978.

6. Feeling overwhelmed. The Stroke Association, Summer 2013.

7. Watkins CL, Wathan JV, Leathley MJ, et al. The 12-month effects of early motivational interviewing after acute stroke: a randomized controlled trial. Stroke. 2011;42(7):1956-1961.

8. Craig P, Dieppe P, Macintyre S, Michie S, Nazareth I, Petticrew M. Developing and evaluating complex interventions: the new Medical Research Council guidance. BMJ. 2008;337:a1655.

9. Waller G. Evidence-based treatment and therapy drift. Behaviour Research and Therapy. 2009;47(2):119-127.

10. Dane AV, Schneider BH. Program integrity in primary and early secondary prevention: are implementation effects out of Control? Clinical Psychology Review. 1998;18(1):23-45. 11. Moyers TB, Martin T, Manuel JK, Hendrickson SM, Miller WR. Assessing competence in the use of motivational interviewing. Journal of Substance Abuse Treatment. 2005;28(1):19-26.

12. Fairburn CG, Cooper Z. Therapist competence, therapy quality, and therapist training. Behaviour Research and Therapy. 2011;49(6-7):373-378.

13. Moore GF, Audrey S, Barker M, et al. Process evaluation of complex interventions: Medical Research Council guidance. BMJ. 2015;350:h1258. 
14. Redfern J, McKevitt C, Wolfe CD. Development of complex interventions in stroke care: a systematic review. Stroke. 2006;37(9)2410-2419.

15. Durlak JA, DuPre EP. Implementation matters: a review of research on the influence of implementation on program outcomes and the factors affecting implementation. American Journal of Community Psychology. 2008;41(3-4):327-350.

16. Mars T, Ellard D, Carnes D, Homer K, Underwood M, Taylor SJC. Fidelity in complex behaviour change interventions: a standardised approach to evaluate intervention integrity. BMJ Open. 2013;3:11.

17. Bumbarger B, Perkins D. After randomised trials: issues related to dissemination of evidence-based interventions. Journal of Children's Services. 2008;3(2):5-64.

18. Goldberg DP, Hillier VF. A scaled version of the General Health Questionnaire. Psychological Medicine. 1979;9(1):139-145.

19. Moyers TB, Martin T, Manuel JK, Miller WR, Ernst D. Revised Global Scales: Motivational Interviewing Treatment Integrity 3.1.1 (MITI 3.1.1). 2010. Unpublished manual.

20. Auton MF, Patel K, Carter B, et al. Motivational interviewing post-stroke: an analysis of stroke survivors' concerns and adjustment. Qualitative Health Research. 2016;26(2):264272.

21. Jelsma JGM, Mertens V-C, Forsberg L, Forsberg L. How to measure motivational interviewing fidelity in randomized controlled trials: practical recommendations.

Contemporary Clinical Trials. 2015;43:93-99.

22. Moyers TB, Rowell LM, Manuel JK, Ernst D, Houck JM. Motivational Interviewing Treatment Integrity Coding Manual 4.2.1: Rational, preliminary reliability and validity. Journal of Substance Abuse Treatment. 2016;65:36-42. 
23. Wade DT, Collin C. The Barthel ADL Index: a standard measure of physical disability? International Disability Studies. 1988;10(2):64-67.

24. Saxon D, Firth N, Barkham M. The relationship between therapist effects and therapy delivery factors: therapy modality, dosage, and non-completion. Administration and Policy in Mental Health. 2017;44:701-715.

25. Miller WR, Rose GS. Toward a theory of motivational interviewing. American Psychologist. 2009;64(6):527-537.

26. Lai DT, Cahill K, Qin Y, Tang JL. Motivational interviewing for smoking cessation. Cochrane Database of Systematic Reviews. 2010;1:CD006936.

27. Firpo-Triplett R, Fuller TR. A Guide to Adapting Evidence-Based Sexual Health Curricula. General Adaptation Guidance, Scotts Valley, CA: ETR Associates/CDC. 2012. 28. Arnow BA, Steidtmann D, Blasé C, et al. 2013. The relationship between the therapeutic alliance and treatment outcome in two distinct psychotherapies for chronic depression. Journal of Consulting and Clinical Psychology. 2013;81(4):627-638.

29. Miller WR, Baca C, Compton WM, et al. Addressing substance abuse in health care settings. Alcoholism: Clinical and Experimental Research. 2006;30(2):292-302.

30. The Health Foundation. Evidence Scan: Training professionals in motivational interviewing. London: The Health Foundation. 2011.

31. Gaume J, Magill M, Longabaugh R, et al. Influence of counselor characteristics and behaviors on the efficacy of a brief motivational intervention for heavy drinking in young men-a randomized controlled trial. Alcoholism: Clinical and Experimental Research. 2014;38(7):2138-2147.

32. Johns RG, Barkham M, Kellett S, Saxon D. A systematic review of therapist effects: A critical narrative update and refinement to Baldwin and Imel's (2013) review. Clinical Psychology Review. 2019;67:78-93. 
33. Moyers TB, Manuel JK, Ernst D. Motivational Interviewing Treatment Integrity Coding Manual 4.2.1. Unpublished manual. 2015.

34. Miller WR, Rollnick S. Motivational Interviewing, Third Edition: Helping People Change Gilford Press. 2012. 
Table 1. Therapist characteristics, motivational interviewing (MI) session factors and outcomes by MI therapist

\begin{tabular}{|c|c|c|c|c|}
\hline Therapist & $\mathbf{A}$ & $\mathbf{B}$ & $\mathbf{C}$ & $\mathbf{D}$ \\
\hline Number of participants allocated & 33 & 81 & 15 & 75 \\
\hline \multicolumn{5}{|l|}{ Therapist Characteristics } \\
\hline Professional background/training & Nursing & Nursing & Psychology & Psychology \\
\hline Stroke knowledge & Extensive & Extensive & Limited & Fair \\
\hline \multicolumn{5}{|l|}{ MI Content $\mathrm{e}^{\mathrm{e}}$} \\
\hline Participants included in analysis (n (\%)) & $12(36)$ & $22^{\mathrm{b}}(27)$ & $4(27)$ & $21(28)$ \\
\hline MI global rating score (median (range)) & $7(6-7)$ & $7(6-7)$ & $7(6-7)$ & $7(6-7)$ \\
\hline MI-consistent utterances ${ }^{\mathrm{a}}(\%)$ & 54 & 52 & 57 & 35 \\
\hline MI-inconsistent utterances ${ }^{\mathrm{a}}(\%)$ & $<1$ & $<1$ & 0 & $<1$ \\
\hline MI-neutral utterances ${ }^{\mathrm{a}}(\%)$ & 46 & 48 & 43 & 65 \\
\hline \multicolumn{5}{|l|}{ MI Dose ${ }^{c}$} \\
\hline Participants completing 4 sessions (n (\%)) & $24(73)$ & $60(74)$ & $7(47)$ & $55(73)$ \\
\hline $\begin{array}{l}\text { Participant's average session length in } \\
\text { minutes (mean (SD)) }\end{array}$ & $43.9(7.4)$ & $36.9(10.9)$ & $47.8(8.1)$ & $29.5(7.7)$ \\
\hline \multicolumn{5}{|l|}{ Outcome $^{\mathrm{d}}$} \\
\hline Participants included in analysis (n (\%)) & $30(91)$ & $66(81)$ & $8(53)$ & $67(89)$ \\
\hline Normal mood at 3 months $(\mathrm{n}(\%))^{\mathrm{f}}$ & $18(60)$ & $41(62)$ & $3(38)$ & $32(48)$ \\
\hline
\end{tabular}

\footnotetext{
${ }^{a}$ Figures are the percentages (rounded) of MI-consistent/inconsistent/neutral utterances across all sessions for the participants included in MI Content analysis

${ }^{\mathrm{b}}$ One participant received MI but was subsequently identified as not having had a stroke so their data were excluded from these analyses

${ }^{c}$ Based on all participants allocated to receive MI

${ }^{d}$ Based on participants included in Outcome analysis

${ }^{\mathrm{e}}$ Assessed using the Motivational Interviewing Treatment Integrity code (MITI 3.1.1) (14)

${ }^{\mathrm{f}}$ Assessed using the GHQ-28 (normal mood: total score <5) (13)
} 
Table 2. Use of selected motivational interviewing (MI) MI-consistent and MI-neutral utterances by MI therapist $\mathrm{t}^{\mathrm{a}}$

\begin{tabular}{lcccc}
\hline Therapist & A & B & C & D \\
\hline Total MI-consistent and MI-neutral utterances & 252 & 473 & 83 & 437 \\
MI-consistent utterances (n (\%)): & & & & \\
Open questions & $25(10)$ & $19(4)$ & $2(2)$ & $31(7)$ \\
Simple reflections & $28(11)$ & $60(13)$ & $25(30)$ & $17(4)$ \\
Complex reflections & $58(23)$ & $116(25)$ & $14(17)$ & $56(13)$ \\
Other MI-consistent utterances & $25(10)$ & $54(11)$ & $7(8)$ & $50(11)$ \\
MI-neutral utterances (n (\%)): & & & & \\
Closed questions & $50(20)$ & $33(7)$ & $22(27)$ & $133(30)$ \\
Other MI-neutral utterances & $66(26)$ & $191(40)$ & $13(16)$ & $150(34)$
\end{tabular}

\footnotetext{
${ }^{a}$ Figures are counts and corresponding percentages (rounded) across all sessions for the participants included in MI Content analysis in table 1
} 